\title{
Insignificant Disease by ECG Finding
}

National Cancer Institute

\section{Source}

National Cancer Institute. Insignificant Disease by ECG Finding. NCI Thesaurus. Code C119244.

An electrocardiographic recording that does not demonstrate any significant signs of ischemia or conduction abnormalities. 